\title{
CHARACTERISATION OF MYCOPLASMA PNEUMONIAE GROWTH FACTORS IN BOVINE SERUM FRACTION
}

\author{
J. H. Hughes, D. C. Thomas, V. V. Hamparian and N. L. Somerson \\ Departments of Medical Microbiology and Pediatrics, The Ohio State University, \\ College of Medicine, and The Children's Hospital Research Foundation, Columbus, \\ Ohio 43205, USA
}

\section{Plates I AND II}

SERUM or a serum component is an essential ingredient of most media used for growing mycoplasmas. This serum requirement can be met by a fraction obtained by ammonium-sulphate precipitation of bovine serum (Smith and Morton, 1951). The bovine-serum fraction (BSF) is now available commercially as PPLO or Mycoplasma Serum Fraction A and can be substituted for whole serum for growing Mycoplasma pneumoniae on glass surfaces (Pollack, Somerson and Senterfit, 1969). During research and development of an experimental M. pneumoniae vaccine (Somerson, 1971; Somerson, Senterfit and Hamparian, 1973) we found that commercially prepared batches of BSF varied in ability to promote growth of the organism on glass. In the present study, we examined these batches by polyacrylamide-gel electrophoresis in an attempt to characterise the factor responsible for the growth-promoting activity.

\section{MATERIALS AND METHODS}

Sources of BSF. Samples of BSF, prepared by the method of Smith and Morton (1951), were obtained from four different sources: Grand Island Biological Company (Grand Island, New York), Microbiological Associates (Bethesda, Maryland), Difco Laboratories (Detroit, Michigan) and Huntingdon Research Center (Baltimore, Maryland). Norman Robillard of Huntingdon Research Center supplied both BSF and a sample of the whole bovine serum from which it had been prepared.

Organism and media. $M$. pneumoniae strain Cl-8 was used. The organism had been isolated from the throat of a 6-year-old boy who presented clinically with primary atypical pneumonia. It was isolated in SSR-5 broth medium, a modification of SSR-4 (Pollack, Somerson and Senterfit, 1970); this differed from the latter medium by the absence of oleic acid and the presence of $3 \%$ and $5 \%$ concentrations respectively of BSF and yeast extract solution. The organism was subcultured and cloned on SSR-5 agar medium, and was identified as $M$. pneumoniae by immunofluorescent and complement-fixing techniques (Somerson et al., 1967; Ertel et al., 1970). After eight subcultures in SSR-5 broth, the Cl-8 strain grew as a confluent layer of organisms attached to the glass surface. It was harvested by discarding the culture fluids and scraping the growth from the glass into a small quantity of SSR-5 broth containing $10 \%$ BSF. After pooling, suspensions were stored, in 1-ml amounts, at $-60^{\circ} \mathrm{C}$. Organisms obtained after the eighth passage on glass were used as inoculum in all experiments.

Photomicrography. For photography of mycoplasmas on glass, the organisms were allowed to grow on coverslips in prescription bottles containing SSR-5 medium. The

Received 4 Apr. 1973; accepted 7 May 1973.

J. MED. MICROBIOL.-VOL. 7 (1974) 


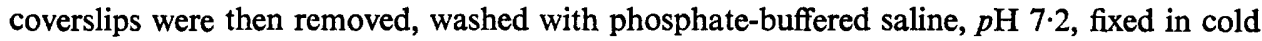
acetone for one min., and stained with Crowle's triple stain (Crowle, 1961) for $5 \mathrm{~min}$. They were rinsed in $2 \%$ acetic acid, dried, and mounted in piccolyte $(60 \%$ solution in xylene). Photographs were taken with a Zeiss Universal Microscope and Nikon Microscope attachment fitted with a Polaroid film back.

Polyacrylamide-gel electrophoresis (PAGE). The techniques were those described by Maizel (1969). Polyacrylamide was recrystallised from chloroform, bisacrylamide from acetone. Five per cent. polyacrylamide gels were prepared by adding to $5 \mathrm{ml}$ of a distilled water solution of acrylamide-bisacrylamide (concentrations of $30 \%$ and $0.8 \%$ respectively) the following materials in sequence: $3 \mathrm{ml}$ of M-sodium phosphate buffer, $0.3 \mathrm{ml}$ of $10 \%$ sodium dodecyl sulphate, $0.015 \mathrm{ml}$ of $\mathrm{N}, \mathrm{N}, \mathrm{N}^{\prime}, \mathrm{N}^{\prime}$-tetramethylethylenediamine (Canalco Industrial Corp., Rockville, Maryland), $21.3 \mathrm{ml}$ of distilled water, and $0.3 \mathrm{ml}$ of $10 \%$ ammonium persulphate. Dissolved gases were removed by suction. The mixture was placed in plexiglass tubes, $4.5 \mathrm{~mm}$ in diameter, and allowed to polymerise. Gel length was $80 \mathrm{~mm}$. Polymerised gels were allowed to stand at room temperature $\left(25^{\circ} \mathrm{C}\right)$ overnight and were stabilised at $5 \mathrm{~mA}$ per gel for one hour; 200- $\mu 1$ samples of BSF (100-200 $\mu \mathrm{g}$ protein), containing $10 \%$ sucrose, were applied to each gel. By means of a Buchler PAGE apparatus, electrophoresis was carried out for 2 hours at $2 \mathrm{~mA}$ per gel followed by $5 \mathrm{~mA}$ per gel for 4 hours. Gels were fixed in 10\% trichloroacetic acid for 18-20 hours and stained for 8 hours with $0.25 \%$ Coomassie brilliant blue. Stain was removed with several changes of $7 \%$ acetic acid either at room temperature or at $40^{\circ} \mathrm{C}$. Gels were scanned in a Gilford Recording Spectrophotometer at $610 \mathrm{~nm}$ for stained gels or at $280 \mathrm{~nm}$ for unstained gels.

Cellulose-acetate electrophoresis. This was done with a clinical electrophoresis system (Millipore Corp, Bedford, Mass.) in barbital buffer, $p \mathrm{H} 8.6$, for $20 \mathrm{~min}$. at 100 volts. The cellulose acetate strips (PhoroSlides, Millipore) were then stained with $1.8 \%$ ponceau-S and decolorised with $5 \%$ acetic acid.

Sephadex-column chromatography. Sephadex G-150 Superfine (Pharmacia Fine Chemicals Inc., Piscataway, New Jersey) was used to separate BSF components. Blue dextran $(0 \cdot 1 \%)$ and phenol red $(0 \cdot 1 \%)$ were used to measure void and bed volumes respectively. Elution was carried out with distilled water at $5^{\circ} \mathrm{C}$. Three-ml fractions were collected in an LKB Ultrarac fraction collector contained in a LKB Calora cold unit. The absorbance of each fraction was determined at $280 \mathrm{~nm}$ in a Gilford Recording Spectrophotometer. The blue dextran eluted from the column in either fraction 13 or 14 (void volume was $39-42 \mathrm{ml}$ ) and the phenol red in fractions $48-50$ (bed volume was $144-150 \mathrm{ml}$ ).

\section{RESULTS}

Assessing growth-promoting activity of BSF. Mycoplasma suspension, $1 \mathrm{ml}$, was inoculated into $25 \mathrm{ml}$ of medium, containing BSF, in 6-oz. prescription bottles. The bottles were incubated at $37^{\circ} \mathrm{C}$ and examined daily for 1 week. To ensure depletion of any serum-fraction nutrients in the inoculum, the growth in each bottle was subcultured. This was done by discarding the culture fluids, suspending the organisms as already described, and inoculating $0.1 \mathrm{ml}$ of the suspension into bottles containing fresh medium. In many cases, bottle surfaces were covered with mycoplasma colonies within 48-72 hours and with a confluent layer of organisms after 4 to 7 days. The quantity of glass-adhering organisms was determined by light microscopy either 1 week after inoculation or when the $p \mathrm{H}$ of the culture fluids dropped to 6.6-6.9.

With some lots of BSF a confluent mass of organisms developed over the entire surface of the bottles. With other batches attachment was irregular, forming isolated "islands" of growth within which the colonies were of very different sizes. A sample of BSF was regarded as having high growth-promoting 
activity when it produced confluent growth of uniformly sized colonies on the glass surface.

$P A G E$ of $B S F$. We wanted to find out why various lots of commercial BSF differed in ability to support mycoplasma growth. PAGE was used to see if this was due to differences in the concentrations of serum components. Electrophoretic patterns of six batches of BSF are shown in fig. 1. Those on the left (A, B, and C) are of batches giving good growth, those on the right (D, $\mathrm{E}$, and F) of batches giving poor attachment and less growth. Three major components were resolved in all six samples. The slow moving component (SMC) was present in highest concentration in the samples with good growthpromoting activity, and it stained with lipid dyes, such as oil red $\sigma$ or Sudan

TABLE

Growth-promoting activity for Mycoplasma pneumoniae of seven samples $(A-G)$ of bovine serum fraction $(B S F)$, and the relative concentrations of three components determined by polyacrylamide-gel electrophoresis

\begin{tabular}{|c|c|c|c|c|}
\hline \multirow{2}{*}{$\begin{array}{l}\text { Bovine serum } \\
\text { fraction (BSF) }\end{array}$} & \multirow{2}{*}{$\begin{array}{c}\text { Growth-promoting } \\
\text { activity } \dagger\end{array}$} & \multicolumn{3}{|c|}{ Quantity of components present in BSF $^{*}$} \\
\hline & & $\begin{array}{c}\text { Slow moving } \\
\text { component (SMC) }\end{array}$ & II & III \\
\hline $\begin{array}{l}\text { A } \\
\text { B } \\
\text { C } \\
\text { D } \\
\text { E } \\
\text { F } \\
\text { G }\end{array}$ & $\begin{array}{l}\text { high } \\
\text { high } \\
\text { high } \\
\text { low } \\
\text { low } \\
\text { low } \\
\text { high: }\end{array}$ & $\begin{array}{c}16(13) \\
14 \\
14(10) \\
3(3) \\
5(5) \\
4(5) \\
8\end{array}$ & $\begin{array}{l}16(12) \\
21 \\
20(11) \\
16(10) \\
19(11) \\
14(11) \\
24\end{array}$ & $\begin{array}{l}68(75) \\
65 \\
66(79) \\
81(87) \\
76(84) \\
82(84) \\
68\end{array}$ \\
\hline
\end{tabular}

* Calculated by scanning stained gels at $610 \mathrm{~nm}$ (or unstained gels at $280 \mathrm{~nm}$ ), and (in parenthesis) expressed as a percentage of whole BSF

$\dagger$ Estimated for $3 \%$ concentrations of BSF in medium.

$\mp$ But less active than fractions A-C.

black B. The other two components (II and III) were present in similar concentrations in all samples and also stained with lipid dyes. From its rate of migration on PAGE, the molecular weight of SMC was calculated as approximately 150,000 daltons.

Using cellulose-acetate electrophoresis we were able to confirm the presence of three components in different batches of BSF. However, we were unable to determine accurately the amounts.

Fig. 2 shows the electrophoretic patterns of a sample of BSF and of the whole bovine serum from which it had been prepared. The same three components found earlier (fig. 1) were also present in the untreated bovine serum, and the SMC was present in relatively large amount. However, the fractionation procedure used in the preparation of the BSF obviously led to loss of significant quantities of SMC; the BSF also had poor growth-promoting activity.

Quantitative correlation of BSF components with biological activity. Two BSF samples, one with high and the other with low biological activity, were 
fractionated by PAGE. The gels were stained and scanned at $610 \mathrm{~nm}$. Fig. 3 shows the relative amounts of the three components in each. The BSF giving the better attachment and growth of mycoplasmas on glass contained the more SMC.

Five additional BSF samples were similarly analysed and the areas occupied by each of the three components, in all seven samples of BSF, were measured with a planimeter and their relative amounts were calculated (table). The BSFs with good biological activity contained the highest concentrations, 8-16\%, of SMC. Conversely, the BSFs with low activity contained much less SMC, $3-4 \%$. There were no obvious differences in the concentrations of components II and III.

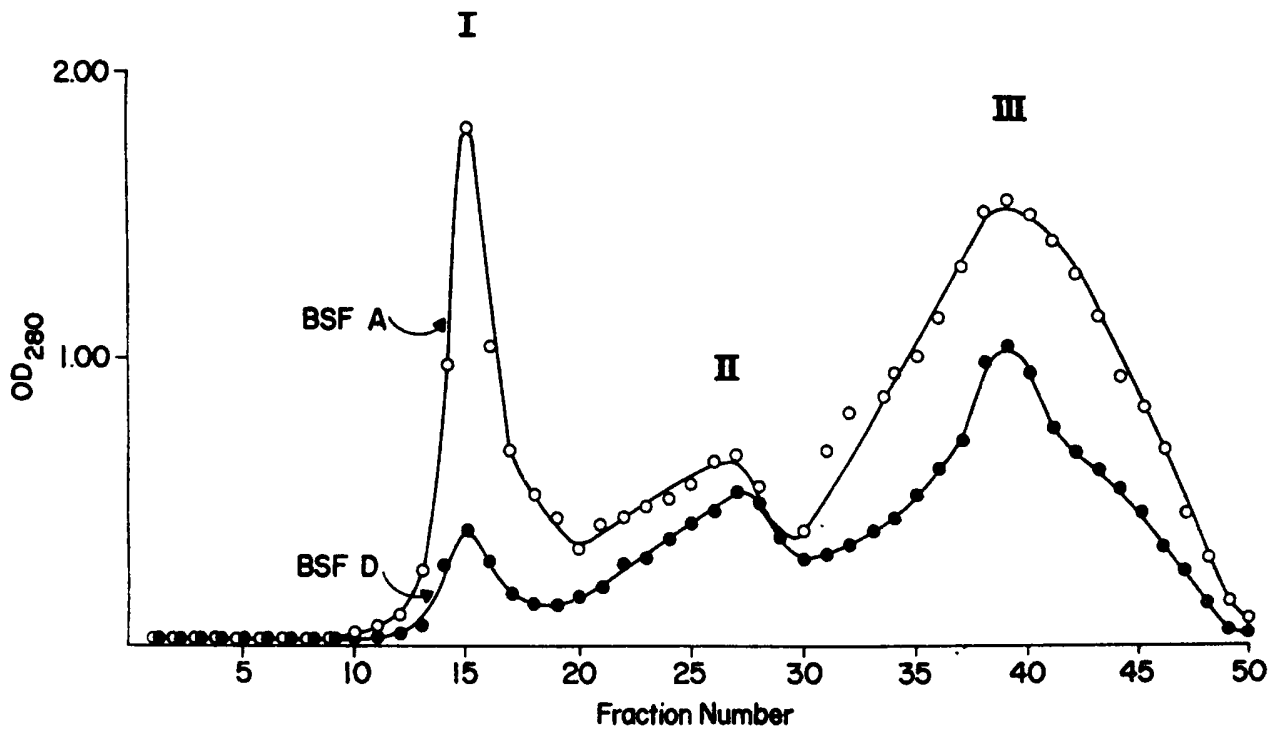

Fig. 4.-Sephadex-column chromatography of two bovine serum fraction batches, BSF-A, with good growth-promoting activity for $M$. pneumoniae, and BSF-D, with poor activity. The elution profiles show the separation of three components, I, II and III.

Sephadex chromatography of BSF. Three components were also resolved on Sephadex G-150. Fig. 4 shows the separation profiles of two samples of BSF, one with high and the other with low biological activity (BSF-A and BSF-D, respectively). BSF-A contained more component I than did BSF-D. Component I corresponds to the SMC of PAGE and eluted from the Sephadex column very close to the void volume. The amount of component II did not differ significantly in the two samples. However, there may have been more component III in sample A than in sample D.

PAGE of components separated by Sephadex chromatography. BSF-B, which gave the same separation pattern as BSF-A (fig. 4), was used for this experiment. The three components were eluted from several Sephadex columns, pooled, and subjected to PAGE; pool I was composed of elution fractions 12 to 18 , pool II of fractions 21 to 29 , and pool III of fractions 31 to 48 . Fig. 5 shows the electrophoresis patterns of BSF-B and its three Sephadex-separated components, 
I, II and III. Apparently, a single fractionation on Sephadex does not completely separate the components. When the three components were mixed in the proportions calculated from whole BSF (table), the electropherogram patterns of the original and the reconsituted BSFs were nearly indistinguishable (fig. 5).

Temperature stability of BSF. When BSF batch A was received in our laboratory, tests showed that it was rich in SMC and had good growth-promoting activity. Growth tests were repeated after storage for 4 months at $-20^{\circ} \mathrm{C}$ or at $4^{\circ} \mathrm{C}$. Mycoplasmas grew sparsely in medium containing BSF that had been stored at $4^{\circ} \mathrm{C}$; they grew much better in the presence of BSF that had been stored at $-20^{\circ} \mathrm{C}$ (fig. 6). SMC was still present, by PAGE, after storage at $4^{\circ} \mathrm{C}$.

\section{Discussion}

Smith and Morton (1951) originally prepared BSF from bovine serum by ammonium-sulphate fractionation. The growth factor was resistant to heat, soluble in trichloracetic acid or chloroform, and was destroyed by proteases, such as trypsin, pepsin, and papain. From their studies with electrophoresis and alumina gels, Smith and Morton (1952) concluded that the growth factor was a low-molecular-weight protein. Subsequently (Smith, Lecce and Lynn, 1954), it was shown to have characteristics in common with a degradation product of $\alpha$-1-lipoprotein, namely in electrophoretic migration activity, sedimentation constant, molecular weight, and lipid composition. Our own finding that SMC stains with lipid dyes supports the conclusion that it is a lipoprotein.

When we used BSF instead of serum for growing M. pneumoniae we got variable results with different batches. The SMC appeared to be the critical factor. When this was present in sufficient quantity the organisms grew well, forming a compact, even, confluent layer on glass. There was excellent correlation between the growth-promoting activity of BSF and its SMC content.

PAGE provided a quick, precise method for determining the amount of SMC in samples of BSF, thus indicating growth-promoting activity. Batches containing less than $5 \%$ SMC were biologically inferior, and those with $10 \%$ or more gave the best results. After storage at $4^{\circ} \mathrm{C}$, the SMC was still present, but apparently in an inactive state. The apparent instability of SMC at $4^{\circ} \mathrm{C}$ may account for the poor growth-promoting activity of some batches of BSF; at present, commercial producers of BSF recommend storage at $4^{\circ} \mathrm{C}$. However, all the BSFs used in our studies were obtained from the manufacturers soon after their preparation and were tested by us for growth-promoting activity shortly afterwards. As we have shown, the processing of BSF from whole serum may significantly reduce the SMC content and this is probably the main reason for variable growth-promoting activity.

We have tested serum from dairy herds and from slaughter-house animals for SMC. Large amounts were detected in samples from both sources, indicating that SMC is probably a normal component of bovine serum. Nevertheless, concentrations of serum as high as $10 \%$, in the medium used by us do not promote good growth and adherence of $M$. pneumoniae. Possibly there are also 
inhibitors in bovine serum that are removed by ammonium sulphate fractionation.

Our present data on SMC applies only to the growth of $M$. pneumoniae on glass. However, a large number of mycoplasma species are cultured in media containing BSF, and SMC may be important for their growth too.

\section{SUMMARY}

Commercially available batches of bovine serum fraction (BSF) vary in their ability to promote the growth of Mycoplasma pneumoniae on glass. Polyacrylamide gel electrophoresis shows the presence in BSF of three major components, which can also be separated by Sephadex chromatography. The slow moving component (SMC) in the gel appears to be the factor responsible for the adherence of $M$. pneumoniae to glass and its growth on that site; batches of BSF containing 6-15\% of SMC possess high activity, those with less than $5 \%$ have low activity. Polyacrylamide gel electrophoresis is a simple and precise technique for determining growth-promoting activity of BSF.

This investigation was supported by Public Health Service contract PH-43-67-78 from the Infectious Disease Branch of The National Institute of Allergy and Infectious Diseases, and by Public Health Service General Research Support grant RR 05504 from The Children's Hospital Research Foundation, Columbus, Ohio 43205.

\section{REFERENCES}

Crowle, A. J. 1961. Immunodiffusion, New York, p. 308.

Ertel, P. Y., ERTel, I. J., Somerson, N. L. and PollaCK, J. D. 1970. Immunofluorescence of mycoplasma colonies grown on coverslips. Proc. Soc. exp. Biol. Med., 134, 441.

MaIzes, J. V., Jr 1969. Acrylamide gel electrophoresis of proteins and nucleic acids. In Fundamental techniques in virology, edited by K. Habel and N. P. Salzman, New York, p. 334.

Pollack, J. D., Somerson, N. L. ANd Senterfit, L. B. 1969. Effect of $p H$ on the immunogenicity of Mycoplasma pneumoniae. J. Bact., 97, 612.

Pollack, J. D., Somerson, N. L. AND Senterfit, L. B. 1970. Isolation, characterization, and immunogenicity of Mycoplasma pneumoniae membranes. Infect. Immun., 2, 326.

SMITH, P. F. AND MoRTon, H. E. 1951. The separation and characterization of the growth factor in serum and ascitic fluid which is required by certain pleuropneumonia-like organisms. J. Bact., 61, 395.

SMith, P. F. AND MorTon, H. E. 1952. Further characterization of the protein factor required by certain pleuropneumonia-like organisms for growth in vitro. Archs Biochem. Biophys., 38, 23.

SMITH, P. F., LECCE, J. G. AND LYNN, R. J. 1954. A lipoprotein as a growth factor for certain pleuropneumonia-like organisms. J. Bact., 68, 627.

Somerson, N. L. 1971. International Conference on the Application of Vaccines against Viral, Rickettsial, and Bacterial Diseases of Man. Pan American Heath Organization Scientific Publication, no. 226, p. 132.

Somerson, N. L., James, W. D., Walls, B. E. ANd Chanock, R. M. 1967. Growth of Mycoplasma pneumoniae on a glass surface. Ann. N.Y. Acad. Sci., 143, 384.

Somerson, N. L., Senterfit, L. B. AND Hamparian, V. V. 1973. Development of a Mycoplasma pneumoniae vaccine. Ann. N.Y. Acad. Sci., 225, 425. 
GROWTH FACTORS FOR $M$. PNEUMONIAE

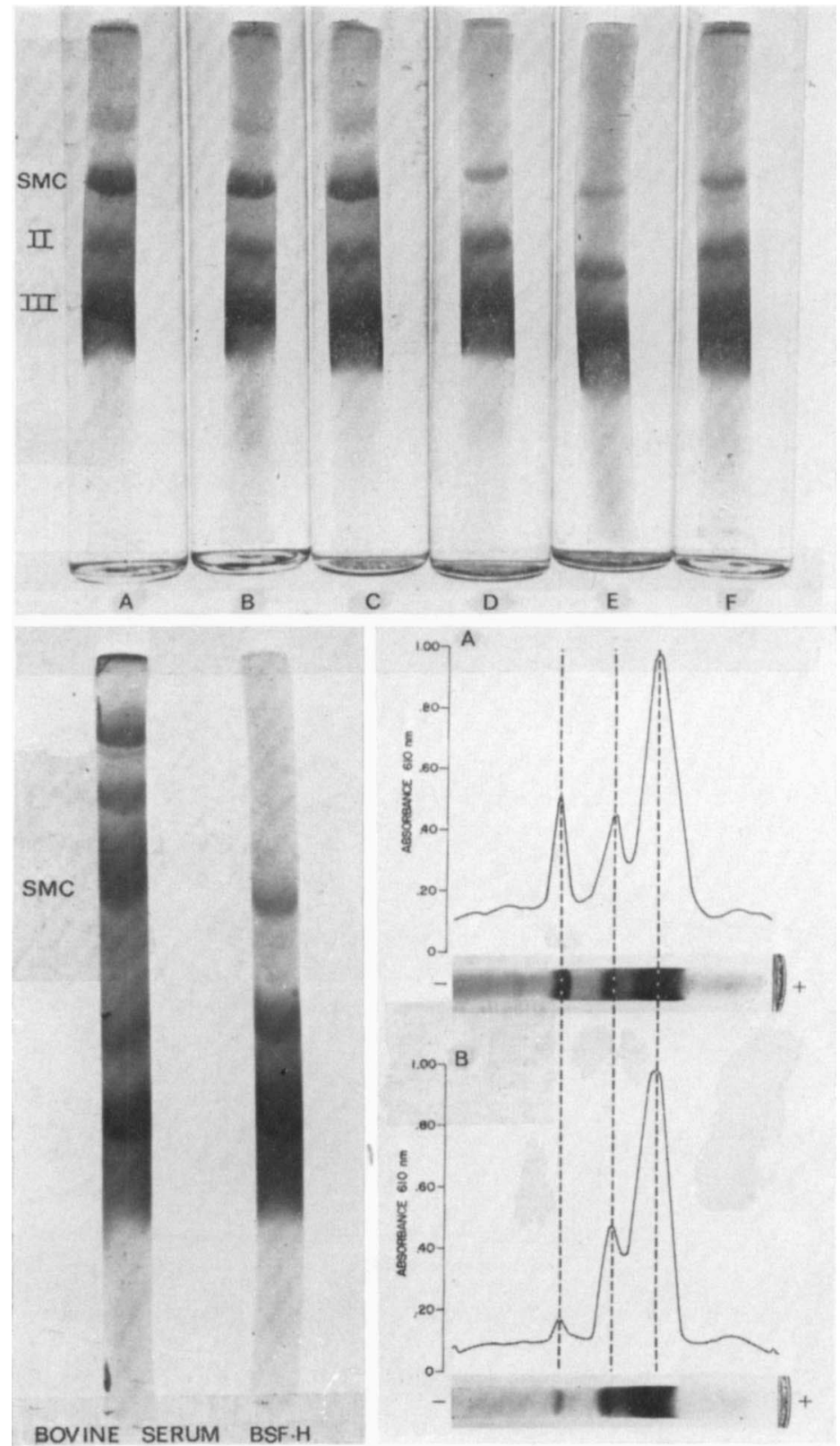

FIG. 1.-Electrophoretic patterns of six different batches (A-F) of bovine serum fraction (BSF). $\mathrm{SMC}=$ slow-moving component.

FIG. 2.-Electrophoretic patterns of bovine serum and bovine-serum fraction (BSF-H) made from it. $\mathrm{SMC}=$ slow moving component.

FIG. 3.-Tracings of polyacrylamide gels scanned at $610 \mathrm{~nm}$. (A) BSF with good growth-promoting activity for Mycoplasma pneumoniae. (B) BSF inadequate for growth of $M$. pneumoniae. 
GROWTH FACTORS FOR $M$. PNEUMONIAE

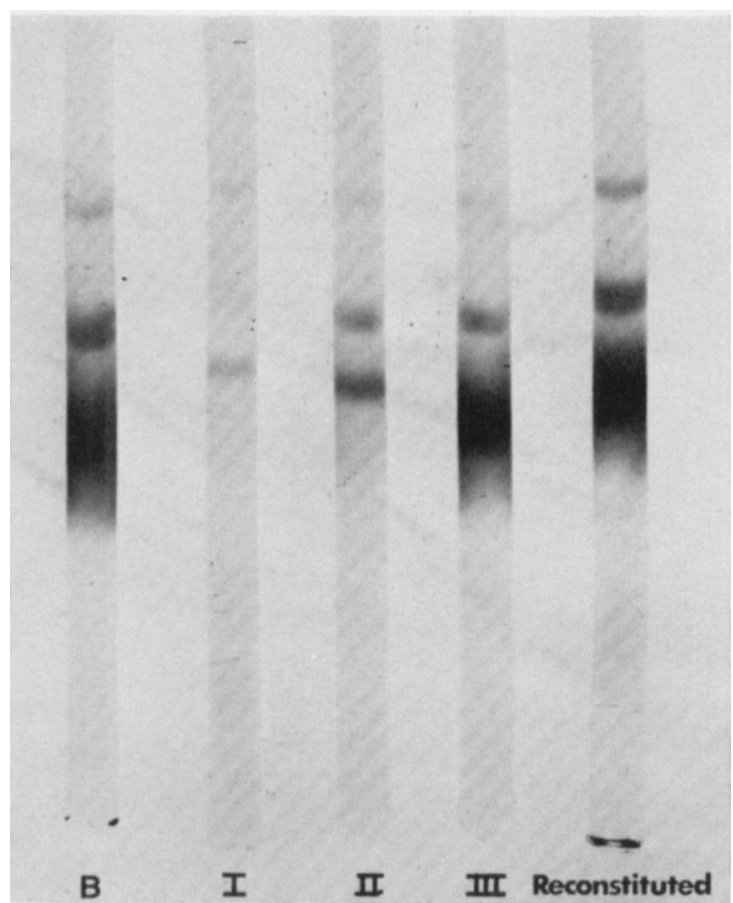

FIG. 5.-Electrophoretic patterns of bovine serum fraction, BSF-B, and of the three components, I, II and III, extracted from it by Sephadex chromatography. The reconstituted pattern was obtained after mixing the three components together in the proportions known to be present in the original sample of BSF.
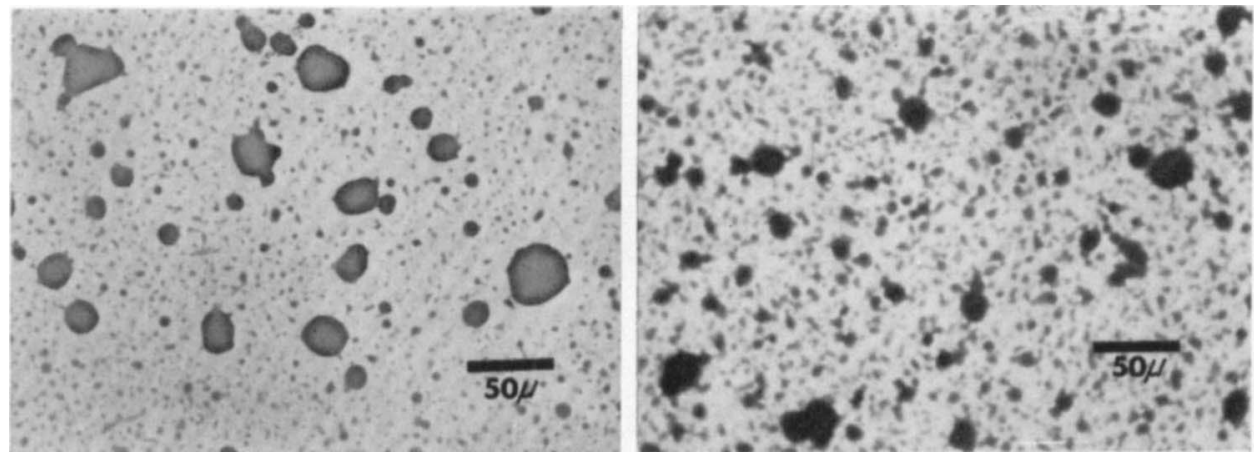

FIG. 6.-Colonies of $M$. pneumoniae grown in medium containing BSF that had been stored at $4^{\circ} \mathrm{C}$ (left) or at $-20^{\circ} \mathrm{C}$ (right) 